\title{
DOI 10.31489/2020No2/39-44
}

UDC $530.1 ; 538.97 ; 539.216 .1 ; 539.216 .2 ; 539.23$

\section{WIDTH OF ENERGY BAND GAP OF NANOPOROUS SEMICONDUCTOR FILMS}

\author{
Zhanabaev Z.Zh., Ikramova S.B., Tileu A.O.,Turlykozhaeva D.A. \\ al-Farabi Kazakh National University, Almaty, Kazakhstan, zhanabaev.zeinulla@list.ru
}

\begin{abstract}
The aim of this work is to experimentally clarify the reasons for the appearance of jumps in the current and memory of semiconductor nanoporous structures.Porous nanostructures were obtained by electrochemical etching. The current-voltage characteristics of the samples were measured for porous silicon and on thin films of a chalcogenide glassy semiconductor. The existence of jump-like switching and current hysteresis in porous silicon nanofilms under laser illumination is shown experimentally.A connection between the switching voltage values and the dependence of the band gap on the porosity of nanofilms is found. These results make it possible to construct a theory of current switching and its hysteresis based on the concepts of the theory of second-order phase transitions.
\end{abstract}

Keywords: porous silicon, energy band gap, current-voltage, electrochemical etching, laser, chaos.

\section{Introduction}

Porous silicon (PS) is a special composite material consisting of pores and silicon crystals. It has a wide range of applications due to the variety of arrangement of surface atoms. The high tunability of porous silicon is determined by many properties, such as dielectric constant [1,2], bandgap [3], etc. The results of work [4-5] showed that porous silicon has a wider energy bandgap $\left(E_{g}\right)$ than solid silicon has. This is explained by the fact that more intense recombination of electron-hole pairs occurs in quantum porous nanostructures and their energy band gaps become larger [6]. In many studies, the band gap is determined through the optical properties (transmission, reflection, absorption) of porous silicon.

The results of work $[7,8]$ showed that the radiation intensity of PS increases with an increase in the percentage of porosity since it is proportional to the number of re-emitted photons. The band gap increased as the concentration of mobile charge carriers in the remaining silicon structure of PS decreased. In this case, the porosity was more than $60 \%$. Energy band gap increments $E_{g}$ in porous silicon are determined from the condition of quantum confinement; therefore, the average size of nanocrystallites is a very important parameter of porous silicon $[9,10]$. It is also noted in $[11,12]$ that with a decrease in the diameter of a porous silicon nanocrystal, $E_{g}$ increases. This is because a decrease in the nanocrystal diameter leads to an increase in the porosity value. In [13] for calculating, the value $E_{g}$ for $\mathrm{Si}$ is calculated by the empiricalpseudo-potential method. It is indicated that the calculation results are in good agreement with other experimental and theoretical results. A linear increase in the bandgap from 1.4 to $1.88 \mathrm{eV}$ with an increase in porosity of PS films in the range of $27-88 \%$ was also noted. This is explained by the arrangement of atoms, mechanical behavior, and quantum confinement of carriers in PS microcrystallites, causing an expansion of the Si bandgap.

In [14], an estimate of the electron energy $\left(E_{g}\right)$ relative to the valence band of bulk Si for nanostructures of oxidized porous silicon was presented. The photoluminescence spectra are explained based on traprelated transitions. For samples with high porosity, double peaks appeared in the PL spectra. One of these peaks remained constant at $\sim 730 \mathrm{~nm}$, while the other was blue-shifted with increasing current density. The PS nanostructure was modeled as an array of regular hexagonal pores, and the average value $E_{g}$ was estimated at $1.67 \mathrm{eV}$. 
The values $E_{g}$ of the zones of n-type and p-type porous silicon layers are larger than $E_{g}=1.11 \mathrm{eV}$ [15]. In the range of porosity (50-76\%), theenergy bandgap of porous p-type silicon (1.80-2.00 $\mathrm{eV}$ ), measured by the optical method,is higher than that of the porous n-type silicon layer (1.70-1.86 eV). For the same porosity, the values $E_{g}$ for p-PSi is always higher than the values $E_{g}$ obtained for the n-PSi layer. The values $E_{g}$ were identified through the transmission spectrum also in [16-17]. From a brief review of the state of the art, it follows that it is important and necessary to study the energy bandwidth of nanoporous semiconductors since it largely depends on the degree of porosity of nanostructures. For example, with an increase in porosity, the values $E_{g}$ increase, the electrical conductivity of nanostructured films changes. Experimental studies currently cover a narrow range of parameters without establishing a universal dependence on their nanoscale structure. More detailed studies are needed to significantly optimize the methodology for experimental studies of nanomaterials to reduce the cost of developing appropriate new technologies.

The aim of this work is to experimentally study dependence of the electrical conductivity of semiconductor nanoporous structures, and effect of the degree of porosity on the value of energy band gap.

\section{Experimental setup and measurement procedure}

Dependence of the energy bandwidth of nanostructured semiconductors on their porosity was investigated experimentally on the surface of porous silicon grown by electrochemical etching. The original silicon was monocrystalline silicon with a $\mathrm{p}-\mathrm{n}+-$ junction, where $\mathrm{n}+$ is a layer, is a diffusion layer with a gradient distribution of phosphorus impurities with a concentration of up to $10^{20} \mathrm{~cm}^{-3}$ with a gradual decrease in impurity to the p-n junction boundary. In the future, the n-layer will be used as a working surface for the formation of porous silicon. The effect of porosity on the conditions of silicon electrochemical etching is investigated.

The thickness of $\mathrm{p}$ - silicon is $350 \mu \mathrm{m}$ with an initial resistivity of $10 \mathrm{Ohm} \cdot \mathrm{cm}$, the thickness of the nlayer is about $500 \mathrm{~nm}$, the surface of the n-layer is mirror-polished. Before electrochemical etching, the silicon surface was etched in a $10 \%$ solution of hydrofluoric acid to remove phosphor silicate glass, thoroughly washed in isopropyl alcohol using an ultrasonic device, and dried.

An electrolyte was used to obtain porous silicon in an HF: ethanol solution in a 1: 1 ratio. The anodic etching was performed in a mode of different current density and anodization time in a specially prepared fluoroplastic cell, and during each etching, the porous silicon layer was thoroughly washed in deionized water and dried.After anodic etching, porous nano- and microcrystalline structures with expanded porous spaces are formed on the surface of crystalline silicon. With an increase in the anodization current density, the porous spaces expand with a decrease in the diameters of silicon clusters. Structural images of nanofilms (Figure 1) were obtained by scanning electron microscopy (SEM) ULTRA 55 FE-SEM (Carl Zeiss).

The porosity was measured by gravimetric weighing. Weighing determined the masses of the sample before anodizing $\left(m_{1}\right)$, after anodizing $\left(m_{2}\right)$, and after removing the porous silicon layer in a $2 \% \mathrm{KOH}$ medium $\left(m_{3}\right)$. Formula

$$
\eta(\%)=\frac{m_{1}-m_{2}}{m_{1}-m_{3}} \times 100 \%
$$

was used.

The experiments on the current-voltage characteristic (CVC) of nanoporous semiconductors were also carried out in the air with laser illumination (infrared laser wavelength $\lambda=630 \mathrm{~nm}, \hbar \omega=0.5 \mathrm{eV}$ ). An n-Si layer with crystal orientation (100) was used as the studied sample. Porous silicon samples with a thickness of $350 \mu \mathrm{m}$ (p-type), $500 \mathrm{~nm}$ (n-type) were obtained by electrochemical etching. The voltage, current density, and taring time were $10 \mathrm{~V}$ and $50 \mathrm{~mA} / \mathrm{cm}^{2}, 3 \mathrm{~min}$, respectively. Metal contacts applied to the n-layer and porous surface (Figure 2) make it possible to obtain the current-voltage characteristic of porous silicon. A voltage was applied to the PC film in the range from $0 \mathrm{~V}$ to $2 \mathrm{~V}$ with a step of $0.1 \mathrm{~V}$ using the Two-Wire Current-Voltage Analyzer of the Ni ELVIS II + universal station. 


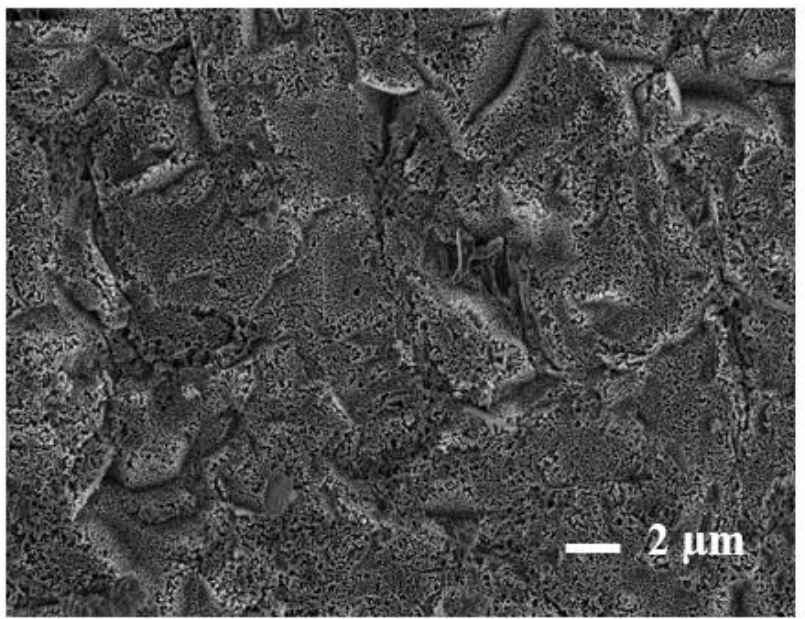

Fig. 1. SEM images of porous silicon layers: top view.

The I - V characteristics of the samples were measured on the NI ELVIS II + educational platform (Figure 2), which uses instruments developed in the LabVIEW software environment on a specially designed prototype board. The main characteristics of the platform: operating current range is $\pm 40 \mathrm{~mA}$, the voltage range is from $-10 \mathrm{~V}$ to $10 \mathrm{~V}$.

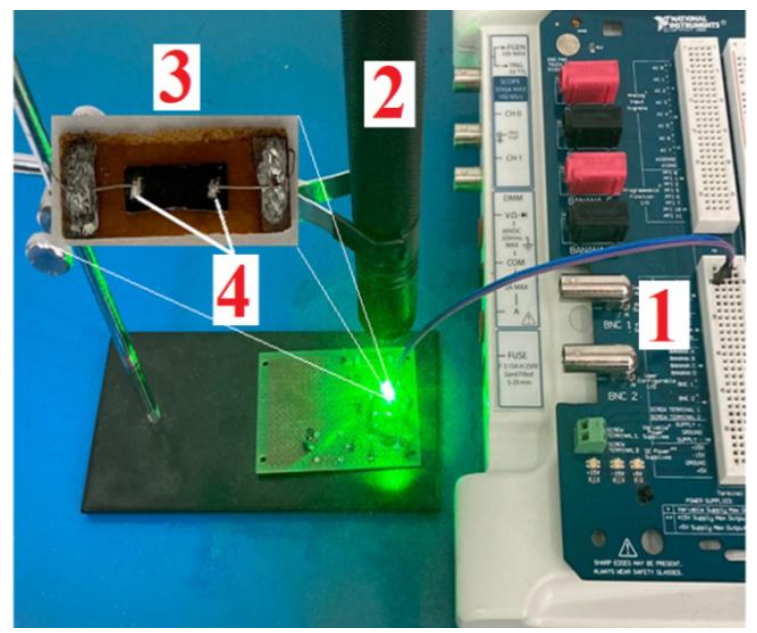

Fig. 2. General view of the installation for measuring the electrical properties of samples: 1- NI ELVIS II ${ }^{+}$platform, 2- infrared laser $\lambda=630 \mathrm{~nm}, 3$-PS films, 4 - contacts.

\section{Electrical conductivity of porous semiconductor nanofilms}

Changes in current versus voltage (VAC) for porous silicon were obtained in natural light (Figure 3) and under laser illumination. The measurements were repeated at regular intervals. Without laser illumination of nanofilms, a jump-like change in current is not observed. Currenthysteresisoccurs.

The reverse current is greater in value than that of the original measurement in the forward direction. This can be explained by a change in the polarization of nanostructures due to the directional action of the primary current.At voltage values of the order of $E_{g}$ and higher (for crystalline silicon $E_{g}=1.12 \mathrm{eV}$ ), currentswitching is observed (Figure 4).At voltage values of the order of $E_{g}$ and higher (for crystalline silicon $E_{g}=1.12 \mathrm{eV}$ ), current switching is observed (Figure 4). 


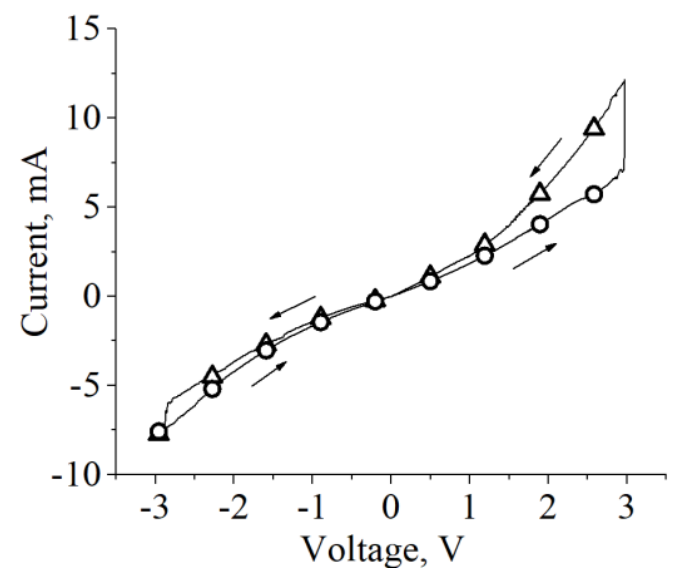

Fig. 3. Current-voltage characteristic of porous silicon in natural light. Measurements in forward $(\Delta)$ and reverse $(0)$ directions.

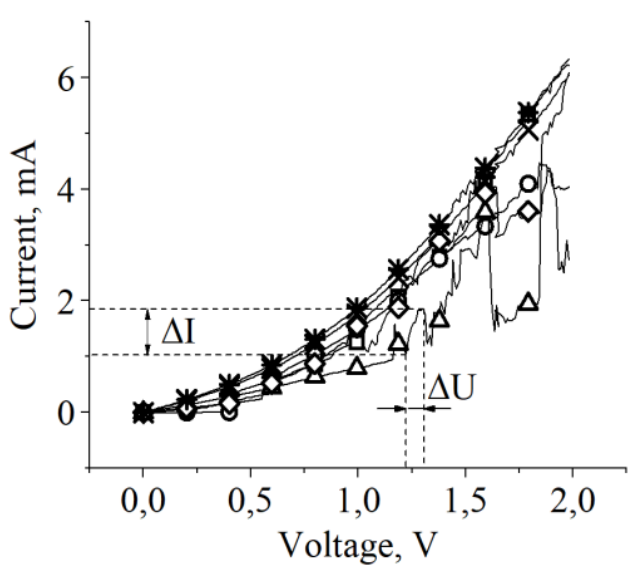

a) measurements at times $t_{0}$

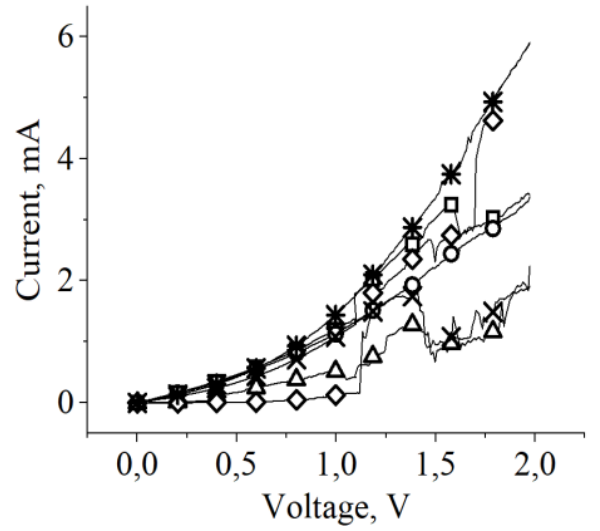

b)after 24 hours

Fig. 4. Volt - ampere characteristics of porous $\mathrm{Si}$ at different time moments under laser illumination of the film.

$$
t_{0}: \mathrm{t}_{0}=0, \mathrm{t}=\mathrm{t}_{0}+\mathrm{n} \tau, \tau=2 \text { min., } \mathrm{n}: \square-0, \circ-2, \Delta-4, \mathrm{x}-6, \diamond-8, *-10 .
$$

The standard deviations were determined by the formulas

$$
x=U / U_{\max }, \sigma_{x}=\sqrt{\left(\left\langle x^{2}\right\rangle-\left(\langle x\rangle^{2}\right)\right)}, y=I / I_{\max }, \sigma_{y}=\sqrt{\left(\left\langle y^{2}\right\rangle-\left(\langle y\rangle^{2}\right)\right)},
$$

where $\left\langle U^{2}\right\rangle=\frac{1}{N} \sum_{i=1}^{N} U_{i}^{2}, N=200$ and are presented in Figure 5.

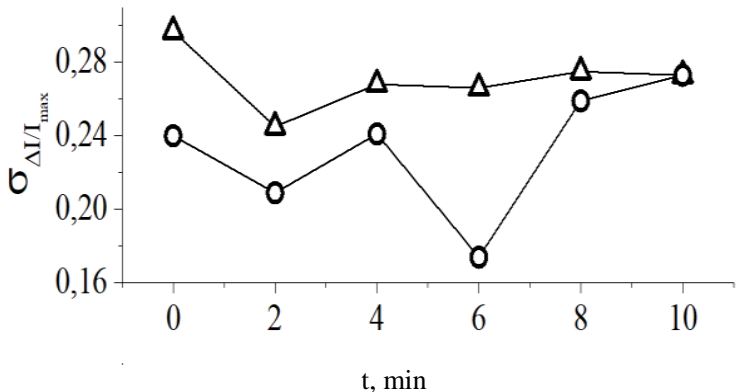

a

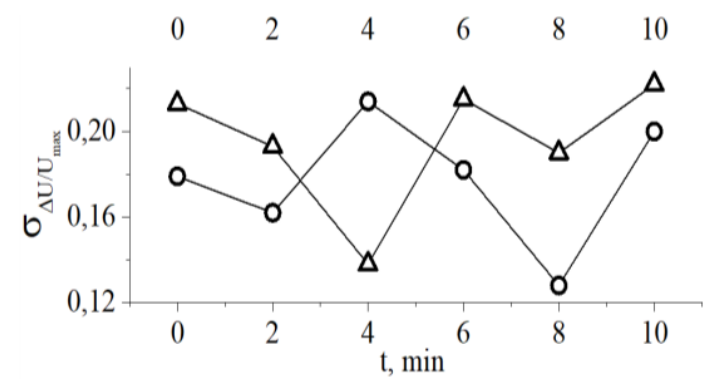

b

Fig.5. Changes in the relative standard deviation of current (a) and voltage (b) over the time sequence of measurements. 
Under laser radiation on nanoporous silicon films, current switching in the region $V \sim E_{g}$ and current hysteresis are also observed. Over time, the hysteresis loop narrows (Figure 6).

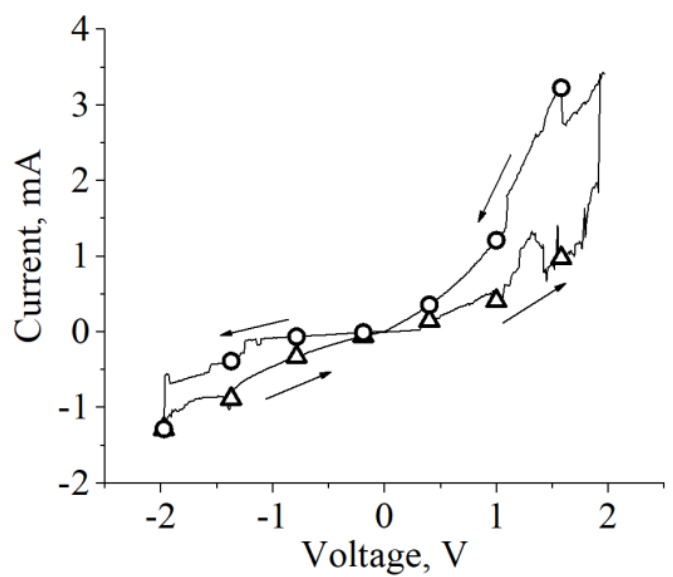

a

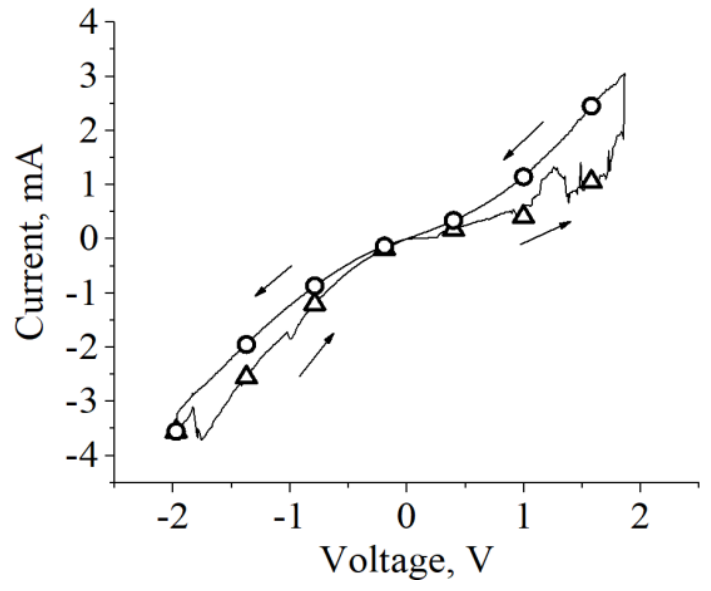

b

Fig.6. Current-voltage characteristic of porous silicon under laser illumination:

a) $\left.-\mathrm{t}_{0}, \mathrm{~b}\right)-\mathrm{t}_{0}+2$ min. $\Delta$ - live measurements, $\mathrm{o}-2$ in the opposite direction.

GST - thin films of a chalcogenide glassy semiconductor of the Ge (germanium) -Sb (antimony) - Te (tellurium) system of composition $\mathrm{Ge}_{2} \mathrm{Sb}_{2} \mathrm{Te}_{2}$, modified with a bismuth impurity. Chalcogenide glassy semiconductors (CGS) have some unique properties and phenomena, such as switching and memory effects, photo structural transformations, and a weak effect of impurities on electronic properties, which have no analogs in crystal materials. The switching effect is observed in thin CGS films and is associated with a reversible stepwise decrease in the CGS resistance in the absence of laser illumination (Figure 7).In Fig.7 $L(\mathrm{~mm})$ - is the distances between the contacts correspond to the numbering of the curves: $1-5$.

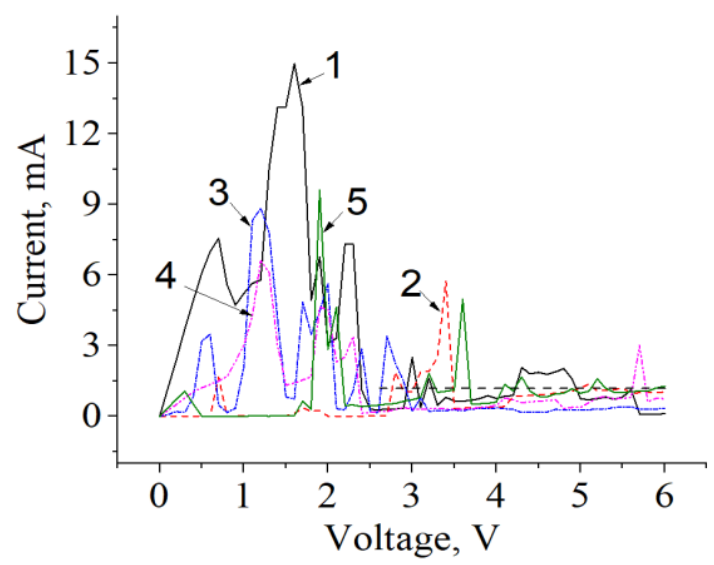

Fig.7. Current-voltage characteristics of GST in natural light.

However, GST films are opaque, non-porous; therefore, their direct use for photovoltaic and gas sensor applications is difficult.

\section{Conclusion}

Porous silicon structures with different diameters are formed by the method of electrochemical anodizing. The current-voltage characteristic of porous silicon has been measured in natural light and under laser radiation. At voltage values of the order of $E_{g}$ and higher (for crystalline silicon $E_{g}=1.12 \mathrm{eV}$ ), switching is observed in the form of an abrupt increase in the current strength. It was found that with an increase in porosity, the width of the energetically forbidden zone of porous silicon increases within 
$\eta \leq 0.8$ This effect is nonlinear since a decrease in $E_{g}$ should be observed at $\eta \rightarrow 1$. Measurements of the electrical characteristics of a non-porous crystal indicate the dependence of $E_{g}$ on the distance between contacts. This fact points to general physical laws between pyroelectrics and nanoporous semiconductors.

\section{REFERENCES}

1 Sun C. Q., Sun X. W., Tay B. K., Lau S. P., Huang H., Li S. Dielectric suppression and its effect on photoabsorption of nanometric semiconductors. Journal of Physics D: Applied Physics, 2001, Vol. 34, No. 15, pp. 2359.

2 Pan L. K., Huang H. T., Sun C.Q. Dielectric relaxation and transition of porous silicon.Journal of applied physics, 2003, Vol. 94, No. 4, pp. 2695-2700.

3 Pan L. K., Sun C. Q. Coordination imperfection enhanced electron-phonon interaction. Journal of applied physics, 2004, Vol. 95, No. 7, pp. 3819-3821.

4 Canham LT. Silicon quantum wire array fabrication by electrochemical and chemical dissolution of wafers.Applied physics letters, 1990, Vol. 57, No. 10, pp. 1046-1048.

5 Hybersen MS. Absorption and emission of light in nanoscale silicon structures.Physical review letters. 1994, Vol. 72, No. 10, pp. 1514.

6 Amri C., OuertaniR., HamdiA., et al. Effect of porous layer engineered with acid vapor etching on optical properties of solid silicon nanowire arrays. Materials \& Design, 2016, Vol. 111, pp. 394-404.

7 Al-Douri Y., Badi N., Voon C. H. Etching time effect on optical properties of porous silicon for solar cells fabrication.Optik, 2017, Vol. 147, pp. 343-349.

8 Abd Rahim A. F., Hashim M. R., Ali N. K.High sensitivity of palladium on porous silicon MSM photodetector.Physica B: Condensed Matter, 2011, Vol. 406, No. 4, pp. 1034-1037.

9 Andersen O.K., Veje E. Experimental study of the energy-band structure of porous silicon.Physical review $B, 1996$, Vol. 53, No. 23, pp. 15643-15651.

10 Wolkin M.V., Jorne J., Fauchet P.M., Allan G., Delerue C. Electronic States and Luminescence in Porous Silicon Quantum Dots. Phys. Rev. Letters, 1999, Vol. 82, No. 1, pp. 197-200.

11 Gaburro Z., Daldossoh N., \&Pavesi L. Porous Silicon.Reference Module in Materials Science and Materials Engineering, 2016, doi:10.1016/b978-0-12-803581-8.01134-6.

12Praveenkumar S. et al.An experimental study of optoelectronic properties of porous silicon for solar cell application.Optik, 2019, Vol. 178, pp. 216-223.

13 Al-Douri Y., Ahmed N.M., Bouarissa N., Bouhemadou A. Investigated optical and elastic properties of Porous silicon: Theoretical study. Materials \& Design, 2011, Vol. 32, No. 7, pp. 4088-4093.

14 Das M. M. et al. Estimation of oxide related electron trap energy of porous silicon nanostructures. Materials Chemistry and Physics, 2010, Vol. 119, No. 3, pp. 524-528.

15 Sheng C. K. et al. Characterization of thermal, optical and carrier transport properties of porous silicon using the photoacoustic technique. Physica B: Condensed Matter, 2008, Vol. 403, No. 17. pp. 2634-2638.

16 Zhang T. T. et al. Cr-doped $\mathrm{BaSnO}_{3}$ nanoporous thin films with tunable band gap via a facile colloidal solution route. Chemical Physics, 2019, Vol. 522. pp. 91-98.

17 Mergen Ö. B., Arda E. Determination of Optical Band Gap Energies of CS/MWCNT Bio-nanocomposites by Tauc and ASF Methods. Synthetic Metals, 2020, Vol. 269, pp. 116539.

Article accepted for publication 03.12.2020 